\title{
Autologous fat grafting and platelet rich plasma (PRP) to treat perioral fibrosis in patients with systemic sclerosis
}

\author{
Miotti GG, Bassetto F, Zocchi M, Brambullo T, Scarpa C and Vindigni V* \\ Vincenzo Vindigni Unit of Plastic and Reconstructive Surgery, Department of Neurosciences, University of Padova, Italy
}

Autologous fat grafting is one of the most used techniques in regenerative medicine surgery. Initially it was just a volumetric technique, with the aim of replacing and filling depressions after injuries, congenital malformations or breast augmentation but later it emerged as a scientifically based regenerative treatment [1]. The regenerative properties are attributed to the presence of adult derived mesenchymal stem cells.

Adipose derived stem cells (ADSCs) can be isolated as a part of the aqueous fraction derived from enzymatic digestion of lipoaspirate (liposuction), the stromal vascular fraction (SVF). SVF is a heterogeneous mesenchymal population of cells that includes not only MSC but also endothelial progenitor cells, immune cells, smooth muscle cells, pericytes, and other stromal components.

Recent advances in the area of tissue regeneration are demonstrating the value of SVF and not only of ADSCs in the regeneration process. That is because SVF works like a network of cytokines, cells and growth factors, with increase propriety of immunomodulation, antiinflammatory, angiogenesis than ADSCs alone [2]. SVF cells can produce several bioactive factors: IGF-1, VEGF, PDGF-BB, MMP-9, b-FGF, Leptin [3].

The major problem of fat grafting is survival rate during time and longstanding results. The literature is in agreement that survival of adipose tissue is unpredictable, with a variable resorption rate $(10 \%$ to $90 \%$ ) [4]. The survival of fat grafts, particularly of a large volume graft, is balanced between this process and hypoxia-induced cell death. These outcomes depend on medical indications, patients' variability and surgical technique. If indications and patients are not modifiable variables, search for the best technique is continuing inspiring important researches into innovation in fat grafting procurement, processing and placement.

A technique to improve the outcomes is to enrich the adipose tissue with platelet-rich-plasma (PRP), another regenerative tool rich in growth factors. The PRP rationale in regenerative medicine comes from the high quantities of growth factors (GFs): the naturally present GFs in PRP may be an ideal resource of nutrients for ADSC and other cells that are threatened by hypoxia and poor vascularization. More so, the combined use of PRP and AT seems to be a great solution to easily combine two regenerative effects. This approach is completely autologous and immediately employed without any type of in vitro preconditioning or media complement.

In-vivo studies have demonstrated that PRP improves the early angiogenesis and revascularization of grafts, increasing survival during time [5].

In particular it shows an important presence of Platelet Derived Growth Factor (PDGF), Transforming Growth Factor-Beta (TGF- $\beta$ ),
Vascular Endothelial Growth Factor (VEGF) and Epithelial Growth Factor (EGF), located into the cytoplasmic alpha-granules and release is triggered by the activation of platelets, promoted by wound environment and by different substances such as thrombin and calcium [6]. GFs are demonstrated to be involved in key stages of wound healing: promoting granulation tissue formation, angiogenesis, matrix formation and remodeling. In addition to growth factors (GFs), platelets release different other substances (e.g. fibronectin, vitronectin, sphingosine 1-phosphate), are extremely important [7]. It is also confirmed that in-vitro PRP can promote migration of amniotic-fluid MSCs and proliferation rate is increased [8].

Speaking about perioral scleroderma, that disease leads both to fibrosis and hypotrophy of perioral sub-cutaneous tissue, and the vascular damage needs an improvement of angiogenesis. Fat grafting technique for perioral fibrosis in SSc is demonstrated by literature consensus to be a good therapy, that relieves symptoms and improves quality of life in patients $[9,10]$. Our group already achieved good results treating perioral fibrosis in SSc with fat grafting technique [11].

With the aim to make results more stable over time and improve even more quality of life, we started the treatment made by fat grafts enriched with PRP in these patients. We are studying them in perspective, making a follow up of 12 months in order to investigate the efficacy of grafts.

The technique consists in an average of $15 \mathrm{cc}$ of purified AT enriched with PRP grafted in the perioral region with $2 \mathrm{~mm}$ blunt cannulas. Access of the injection are localized in the angle of labial commissure and from that access equal parts of the composite graft are injected in the subdermal tissues and submucosally in each patient to both superior and inferior hemi-labial regions (Figure 1).

The follow up is composed by a subjective questionnaire, ultrasound scans and mouth diameter measurements. The questionnaire is administered at 6- and 12-months post-op, in which patients were asked to evaluate variations in functional and aesthetic aspects relative to the perioral region.

The perioral region is assessed by ultrasound scans preoperatively, at 6 and 12 months post-operatively at 4 standard points (canine eminence, $1 \mathrm{~cm}$ lateral to the labial angle, median $1 \mathrm{~cm}$ below the lower

${ }^{*}$ Correspondence to: Vincenzo Vindigni, Unit of Plastic and Reconstructive Surgery, Department of Neurosciences, University of Padova, Italy, E-mail: vincenzo.vindigni@unipd.it

Received: January 14, 2020; Accepted: January 24, 2020; Published: January 27, 2020 


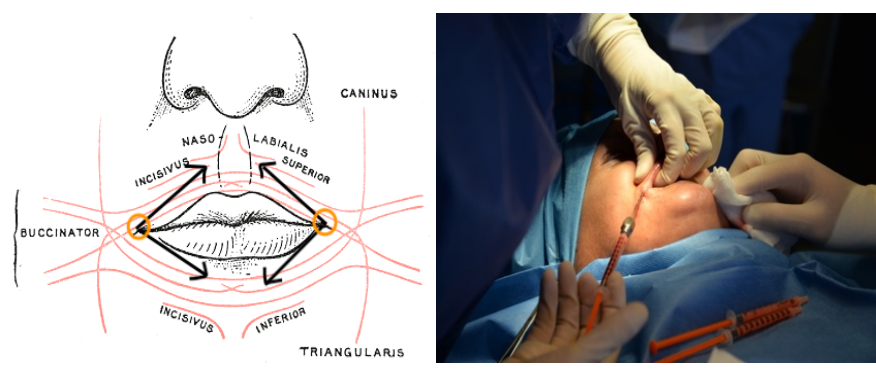

Figure 1. Accesses and sites of injection

lip vermillion, median at the mental bony eminence), to assess the outcome of grafts.

More, we measure at pre-operative time, at 6 months and 12 months the vertical and lateral diameters as linear distances between inner mucosal borders of the orifices. The same surgical procedure is proposed again to the patient at the end of the follow up.

Nowadays we are continuing the follow-up and surgical procedures. Results obtained are promising: all patients treated reported their full satisfaction with the operation and all patients stated they would repeat the operation or suggest it to others. The measurements performed are showing an increase both of subcutaneous thickness and mouth diameters.

Observing our experience, composite graft seems to improve fat maintenance during time and regeneration of tissues. This may mean that patients can have more benefit for longer time. These conclusions require several years of follow up and may depend on how many operations our performed on the same patient.

On the basis of these results we agree with the idea that the regenerative effect is the result of a set of elements:

- Adult adipocytes which restore a sliding softer layer and improve mechanical qualities of tissue

- Platelet growth factors promotes granulation tissue and angiogenesis

- Immunomodulatory effects both of the ADSC and PRP, bringing a reduction of the inflammatory response
- ADSCs naturally present in the AT that can differentiate

- GFs that promote the proliferation of ADSC and other cells and modulate the fibroblasts activity

\section{Conclusion}

Our future challenge is to introduce in our follow-up a method for assessing histological and molecular change: clinical activity and macro objectives evidences are nowadays little without a laboratory confirmation which investigates the micro ones, especially in regenerative medicine.

\section{References}

1. Coleman SR (2006) Structural fat grafting: More than a permanent filler. Plast Reconstr Surg 118: 108S-120S.

2. Charles-de-Sá L, Gontijo-de-Amorim NF, Maeda Takiya C (2015) Antiaging treatment of the facial skin by fat graft and adipose-derived stem cells. Plast Reconstr Surg 135: 999-1009.

3. Traktuev DO, Prater DN, Merfeld-Clauss S (2009) Robust functional vascular network formation in vivo by cooperation of adipose progenitor and endothelial cells. Circ Res 104: $1410-1420$

4. Ross RJ, Shayan R, Mutimer KL, Ashton MW (2014) Autologous Fat Grafting: Curren state of the art and critical review. Ann Plast Surg 73: 352-357.

5. Nakamura S, Ishihara M, Takikawa M (2010) Platelet-rich plasma (PRP) promotes survival of fat-grafts in rats. Ann Plast Surg 65: 101-106.

6. Martineau I, Lacoste E, Gagnon G (2004) Effects of calcium and thrombin on growth factor release from platelet concentrates: Kinetics and regulation of endothelial cell proliferation. Biomaterials 25: 4489-4502.

7. Kaur P, Puneet, Dahiya V (2011) Platelet-rich plasma: A Novel bioengineering concept Trends Biomater Artif Organs 25: 86-90.

8. Roubelakis MG, Trohatou O, Roubelakis A (2014) Platelet-rich plasma (PRP) promotes fetal mesenchymal stem/stromal cell migration and wound healing process. Stem Cell Rev Rep 10: 417-428.

9. Magalon G, Daumas A, Sautereau N, Magalon J, Sabatier F, et al. (2015) Regenerative approach to scleroderma with fat grafting. Clin Plast Surg 42: 353-364.

10. Del Papa N, Caviggioli F, Sambataro D (2015) Autologous fat grafting in the treatmen of fibrotic perioral changes in patients with systemic sclerosis. Cell Transplant 24: 6372 .

11. Scarpa C, Rizzo M, Vezzaro R (2019) Greffe de graisse dans la sclérodermie périorale : une série de cas. Rev Francoph Cicatrisation 3: 46-52.

Copyright: (C2020 Miotti GG. This is an open-access article distributed under the terms of the Creative Commons Attribution License, which permits unrestricted use, distribution, and reproduction in any medium, provided the original author and source are credited. 